\title{
Technology Development Can Lead to Smaller Mars Ascent Vehicles, for Multiple Affordable Sample Returns
}

\author{
John Whitehead
}

$530-758-8115$

Consultant

john.whitehead@alumni.caltech.edu

A white paper submitted for the Decadal Survey on Planetary Science and Astrobiology

2020 July 12 (third of three by this author)

Revised with minor corrections, clarifications, and some additions

Resubmitted on 2020 September 11 


\section{$\underline{\text { Introduction }}$}

New technology development can lead to smaller Mars ascent vehicles (MAVs), so that multiple Mars Sample Return (MSR) missions will become affordable. As is true for Earth launch vehicles, pump-fed liquid propulsion offers the most long-term promise, because lightweight rocket parts let smaller vehicles lift heavier payloads. Rocket engine pumps enable low tank pressures for low tank mass, while high pressures in combustion chambers and rocket nozzles let these parts be smaller, for less mass. The pump-fed rocket principle has been demonstrated on a small scale, but not refined and used in space, because satellites and traditional planetary missions do not need miniature launch vehicles, like MSR does. A new kind of pump is needed.

In order to implement pump-fed rocket propulsion small enough for a MAV, dedicated development efforts are needed. The latter has not occurred, at least partly because misconceptions support hopes for an easy path to a MAV. Ironically, over the course of 40 years of expectations for MSR, there has never really been a long-term effort for MAV technology development. A few miniature rocket engine pumps have been built and tested, but such efforts have been sporadic and isolated. Ideally there would be an established community of specialists to advocate for funding and to participate in peer review.

This white paper discusses the quest for a smaller MAV, and the innovation dilemma. Existing small-scale rocket technologies are then described, along with the challenges for making a MAV that is significantly smaller (e.g. $200 \mathrm{~kg}$ ) than is presently envisioned (400 kg). Possibilities for miniaturizing pump-fed liquid propulsion are reviewed.

\section{Seeking a Smaller MAV}

In the late 1990's, MSR was expected to be accomplished more than once before the year 2010, using science rovers that would each carry a small MAV for sending geology samples to Mars orbit. More than two decades later, long-awaited MSR plans have converged toward one future flagship-class Mars lander that will deliver not primarily science instruments, but rather a large 400-kg MAV plus its support equipment, and a fetch rover to gather sample tubes left by the Mars 2020 rover (Perseverance). A primary reason for the postponement, and the reconfigured mission architecture, is that a small MAV has remained beyond reach.

While MSR becomes more affordable if the MAV can be smaller, no one really knows how small a MAV can be, or the achievable payload mass. Numerous design studies and propulsion research efforts have been inconclusive, which points to a need for innovation. A MAV needs to be a miniature launch vehicle, with far more propulsive capability than a satellite or a spacecraft, in terms of both thrust-to-weight and velocity change. The MAV challenge includes overcoming cultural norms inherent to conventional practices for small-scale space propulsion. 


\section{The Innovation Dilemma}

For good reasons, propulsion engineering for satellites and planetary spacecraft is dominated by a conservative culture of risk avoidance. By design, system functionality is little more than the sum of component functionality, so that complete propulsion systems do not need testing before flight. Reliable operation is ensured by including redundant components (spare parts connected and ready), and by minimizing deviations from component designs previously proven in space. The lexicon of space propulsion emphasizes words such as "heritage," "pedigree," "mature," and "select." The latter word is used in the sense that new propulsion systems are made of parts that are selected, avoiding new designs whenever possible.

Whether the smallest MAV will be $400 \mathrm{~kg}$ or $200 \mathrm{~kg}$, there will be custom-designed components with little redundancy, and complete system testing will be worthwhile. To the extent that innovation can be more fully embraced, somewhat contrary to the established culture of space propulsion engineering, there can be progress toward a smaller MAV. Greater progress needs original ideas with trial-and-error testing, guided by underlying physical principles. The most important physical principle is simply conservation of momentum, because rockets are momentum machines that reach higher speeds (or carry more payload) if the mass of components is less, relative to both the propellant mass and thrust forces.

For Earth departure, launch vehicle engineering is a balancing act that requires detailed analysis and careful testing to refine every piece, resulting in rocket stages that are over 90 percent propellant. Extra structural mass reduces flight performance, while structural failures and explosions result from parts made too lightweight. Examples of innovation for Earth launch vehicles are given below. Impressive new capabilities have been achieved in recent years. One ingredient for success is sustained efforts that take longer than building a satellite propulsion system. A related ingredient is a tolerance for disappointing test failures.

The SpaceX Falcon 9 first stage has enough extra propulsive capability to include landing legs and return intact for re-use, a feat unexpected in 2010. On the second stage, the total mass of parts is so low that the vehicle does well with kerosene fuel, despite the lower exhaust velocity compared to hydrogen fuel traditionally used for upper stages. Loading super-cold propellants at the last minute, with people on board, was considered too risky a few years ago.

The Electron small launch vehicle, developed by Rocket Lab in recent years, is another example of creative rocket engineering [1]. While big rocket engines have turbines to spin centrifugal pumps, the Electron uses electric motors to turn its pumps, without being overburdened by battery weight, as could easily have happened. The smallest Earth launch vehicles have traditionally used solid propellant, e.g. Scout (17 tons) and Pegasus (18.5 tons). The Electron is less massive (12.5 tons), so it has set a new standard as a smaller operational launch vehicle, while providing a shining example of scaling down pump-fed liquid propulsion. 
In 2018, a team in Japan set a record for the smallest rocket to ever send a satellite to Earth orbit [2]. Only 2.6 tons total at launch, the payload fraction was 0.12 percent, a $3 U$ cubesat. While not an operational launch vehicle, the one-time success of the SS-520-5 offers an excellent example of innovation, with special efforts to make lightweight components. Complete guidance, with continuous thrust vector steering, would have made the rocket parts too heavy relative to the propellant mass. All three stages were spinning during flight, which averages out thrust vector misalignments while also maintaining a steady direction by virtue of the angular momentum vector. The first flight test failed, but provided useful data through telemetry, illustrating the value of trial-and-error testing in cutting-edge engineering.

The above examples show that new advances do happen for launch challenges. MAV development can almost certainly benefit from a similar innovation culture, with emphasis on building lightweight components and flight testing complete MAV designs.

\section{Overview of Small-Scale Liquid Rocket Propulsion}

Small-scale rocket propulsion, widely implemented for satellite and spacecraft maneuvers, differs greatly from launch vehicle propulsion. Simplicity is of paramount importance, for the sake of long-term reliability (multiple maneuvers over many years). Redundancy is typical, hence extra component mass. As noted above, the use of previously flight-proven component designs is so highly valued that avoiding innovation at the component level can be a source of pride. Unique new systems are proudly designed, built, and flown within such constraints.

On satellites and planetary spacecraft, liquid propulsion systems use stored inert gas to displace propellants from their tanks, through control valves, then into combustion chambers. In order for propellants to flow, liquid tank pressures (hundreds of psi) must exceed combustion chamber pressures. Helium gas is often carried in storage vessels at much higher pressures (thousands of psi). This type of system is known as pressure-fed propulsion (no pumps).

For structural integrity, tank wall thickness, hence mass relative to propellant volume, increases with pressure, so low tank pressures are desirable. However, low pressure in a combustion chamber requires the chamber and exhaust nozzle to be relatively large, and therefore heavy relative to thrust. Considering that tanks can be lighter if engines are heavier and vice-versa, preferred pressure levels depend on mission maneuvering requirements.

Most satellites and spacecraft have big tanks and relatively low thrust, for high velocity changes done gradually. Tank mass dominates, so lower pressures reduce total component mass, despite combustion chambers being heavy relative to thrust. For science at Saturn, more than half of the 5.6-ton starting mass of the Cassini spacecraft was 3 tons of liquid propellants below 250 psi, while the thrust-to-mass ratio was only about one percent of Earth gravity [3]. Hundreds of satellites in geostationary Earth orbit started with a similar liquid fraction, to 
perform about $2500 \mathrm{~m} / \mathrm{s}$ of velocity change maneuvers. That includes roughly $1750 \mathrm{~m} / \mathrm{s}$ for orbit insertion, followed by $50 \mathrm{~m} / \mathrm{s}$ each year to correct orbit drift over a 15-year life. Such propulsion systems, without the rest of the spacecraft, could theoretically achieve Mars' orbit velocity, but they would not be able to lift themselves in Mars gravity. Adding thrust chamber mass would detract from the velocity.

If a small velocity change is needed in a short time (high thrust-to-mass ratio), then thrust chamber mass dominates tank mass, so higher pressures can reduce total component mass. For example, landing on Mars needs a high thrust-to-mass ratio to overcome gravity, but the rocket velocity change for landing is only about one tenth of orbit velocity. The sky crane Mars landing system feeds relatively compact thrust chambers from hydrazine tanks above 700 psi [4]. The tanks are relatively small and therefore not unduly heavy, despite having wall thickness for this unusually high pressure.

Mars ascent needs high thrust-to-weight and high velocity together, but pressure-fed operation would require tanks and thrust chambers to both be relatively heavy. It is not practical to build a MAV by assembling components made for satellites [5]. In theory, pressure-fed MAV propulsion might be enabled by all-new custom components, e.g. made of improved structural materials having a higher ratio of strength to mass density.

\section{Solid Rocket Propulsion on a Small Scale}

Small solid rocket motors (SRMs) have been used for spacecraft maneuvers (by convention, "rocket motor" indicates solid propellants, while "rocket engine" indicates liquid propellants). Typically, an SRM performs one major maneuver all at once, burning its intimate mixture of oxidizer and fuel until all the propellant is gone. SRMs have been used for entering planetary orbits (Venus Radar Mapper, "Magellan," 1990), and for lunar landing (Surveyor, 1966-1968). In both of these examples, the SRM was attached to a spacecraft that used liquid propulsion for directional control. The Surveyor SRM cancelled almost all of the incoming velocity, then was jettisoned before the liquid propulsion system completed the soft landing.

SRMs for space maneuvers are a highly refined, mature technology. For sizes like a MAV needs, the non-propellant mass is very low. It is very tempting to assume that a solid propellant MAV can be readily built. However, adding a steering system can easily make the component mass too heavy. For perspective, small military missiles use solid rocket motors plus steering systems, and they would not be able to launch from Mars to orbit.

NASA has begun to develop a two-stage solid propellant MAV [6, 7], intended to depart from Earth as early as 2026. Propulsion technical publications indicate that challenges lie ahead, to meet a 400-kg mass limit [8-11]. The total component mass needed for nozzle steering remains uncertain. After the first stage burn, the upper stage needs liquid propulsion for steering 
during a long coast phase. The necessary amount of liquid propellant is uncertain, because the coast phase might begin at a low altitude, resulting in unpredictable aerodynamic disturbances [12]. Reduced thrust over a longer burn time will shorten the coast time in the atmosphere, reduce aerodynamic drag, and permit a smaller SRM nozzle with lighter steering components. Ideally, the solid propellant can burn much slower than in proven SRMs, not easily done.

Assuming that a solid propellant MAV can be built, it will be much harder to scale it down, because smaller solid rocket motors naturally have even shorter burn times, with thrust-toweight ratios even farther in excess of what a MAV needs. Given more excess acceleration, any steering system becomes relatively heavier as a solid MAV is scaled down, and the detrimental effect of aerodynamic drag increases due to more excess speed lower in the Mars atmosphere [13]. A liquid propellant attitude control system for the coast phase would also need to be scaled down, resulting in heavier components relative to the propellant mass. A solid propellant MAV might be less heavy if liquid is pumped in its steering subsystem (see below).

In the late 1990 's, spin stabilization of solid rocket stages was suggested as a way to develop the smallest possible MAV ( $<100 \mathrm{~kg})$, by avoiding the mass of parts for active directional control including inertial measurement and steering components [14]. Future work toward such a MAV might be inspired by the SS-520-5 achievement in Japan, described above. The latter rocket had heavy ground support equipment, a rigid launch rail to point it at the correct angle. Fins were used to aerodynamically initiate rotation in Earth's thick atmosphere. A similar launch from Mars might need a heavy rotating spin table, rigidly fixed to the ground, and adjusted to an appropriate angle depending on wind. Wind measurements would need additional support equipment to be sent to Mars. In the absence of active guidance, inaccuracies in the final orbit would have to be taken into consideration for rendezvous with the earth return orbiter.

\section{Hybrid Rocket Propulsion}

Hybrid rocket propulsion uses a liquid oxidizer to burn a solid fuel (not a solid propellant as in SRMs). A hybrid MAV was researched by JPL and MSFC in recent years, because the fuel would potentially withstand low temperatures on Mars, better than SRM propellant [15]. Remaining unknowns include propellant challenges, e.g. thermal expansion and reliable ignition [16]. Some components would likely be heavy, notably tanks for oxidizer and helium. NASA noted a mass reduction would result from using a low-pressure oxidizer tank, with a pump [17].

\section{Miniaturizing Pump-Fed Liquid Rocket Propulsion}

The thrust force of solid rockets and hybrid rockets depends on the amount of propellant, due to a natural tendency for an exposed surface to burn at a particular rate. In contrast, the thrust of a liquid rocket is set by the engine size, which is independent of the propellant quantity (tank sizing). Therefore, the desired acceleration (thrust to mass ratio) can be set independently 
from the total velocity change required to reach orbit. A liquid propellant MAV can accelerate slowly enough to avoid high aerodynamic drag, and continue burning until the Mars atmospheric density becomes negligible [13].

A smaller MAV is beyond spacecraft technology, so it is worth asking how launching from Earth is done. Large rocket engines use turbines powered by high-temperature gases (from combustion) to drive centrifugal pumps. Pump-fed rocket engines are complicated, so they would not be used if not essential. The benefit of pumps is to let tanks be very lightweight by virtue of low pressures ( $<50 \mathrm{psi}$ ), while permitting combustion chambers and nozzles to be lightweight by virtue of being compact, enabled by high pressures (> 500 psi, often $>1000$ psi). Low pressures for the liquid tanks avoid the kind of inert gas storage used for satellite propulsion, an additional mass savings considering fewer or smaller pressure vessels.

Ideally, a MAV would use the above principle on a very small scale, for which there are multiple challenges. In order for tank mass to benefit from low pressure, the walls need to be quite thin $(<0.5 \mathrm{~mm})$, potentially delicate. Small high-pressure thrust chambers need to run for minutes without overheating. Miniature pumps and associated parts (valves, etc.) must be significantly less heavy than the net mass advantage realized by lightweight tanks and thrust chambers.

In 2009, the lead NASA engineer for MAV propulsion development convened an expert panel to analyze multiple possibilities. They concluded that a single-stage pump-fed MAV would be significantly smaller, compared to a two-stage solid rocket and a pressure-fed MAV [18]. The solid propellant MAV received the highest overall rating, due to fewer unknowns. The pumpfed option was ranked only second, essentially due to the expectation that MSR would happen too soon thereafter to allow time for a development effort. Now another decade has passed.

In 2015, a JPL study also concluded that a pump-fed liquid MAV would be less massive than solid options, and less massive than a pressure-fed liquid MAV [19]. The same study favored hybrid propulsion, which after years of funded testing is now less favored than the two-stage solid [6]. While a well-designed pump-fed MAV should be much better than any other option, the 2015 study found a relatively small advantage, likely because it was assumed that the pumps would be powered by electric motors with heavy batteries [17]. A 2014 version of the same JPL study similarly ranked a pump-fed MAV as preferred over solid propellant and pressure-fed options [20]. This reference noted that small turbines would not work very well, so rotating pumps would be powered by electric motors.

Both JPL and Rocket Lab concluded that batteries and electric motors are better than small turbines, for spinning small centrifugal pumps. Sutton noted that the smallest centrifugal pumps are "notoriously inefficient," but positive displacement pumps can be much better for small rockets [21]. This same book describes a few instances of rocket flights using positive displacement pumps, including a proof-of-principle test flight of a pump-fed rocket launched 
from Vandenberg in 1994 [22]. A four-chamber piston pump had one-inch diameter cylinder bores that received hydrazine propellant at $50 \mathrm{psi}$ and delivered it to thrust chambers at over ten times the tank pressure. A small fraction of the pumped propellant was reacted to make high temperature gas that powered the pump, avoiding motors and battery mass.

The 1994 pump design was later refined somewhat. In 2005-2006, it was tested for the NASA Mars Program [23]. The operating lifetime was almost long enough for a MAV. There was no dedicated funding directly related to MSR, so the effort did not continue at that time. Pumpfed liquid rocket propulsion for a MAV remains an open question, worthy of future creative engineering efforts. The type of pumps used for Earth launch do not work well, or not at all, if small enough for a MAV. Possibilities remain unexplored, for positive displacement pumps.

In conclusion, multiple NASA publications have stated that pump-fed liquid propulsion can result in a smaller MAV, and some limited testing has shown promise. The possibilities have not been vigorously pursued, partly because the culture of space propulsion tends to shy away from new technology, partly because most other missions don't need MAV technology, and partly because of the notion that a MAV can readily be developed in only a few short years after MSR mission plans are in place. After 40 years of hopes for MSR, the MAV is still a missing link, so it would be a good idea to fund long-term MAV development, regardless of MSR mission plans. Future work needs to be guided primarily by the mass savings that can be realized, instead of by near-term schedule urgency or by the notion that propellant selection readily leads to a final MAV design.

\section{$\underline{\text { References cited }}$}

1. Tulp, J., and Beck, P., "Rocket Lab: Liberating the Small Satellite Market," 31st Annual Small Satellite Conference, SSC17-IV-04, August 2017. https://digitalcommons.usu.edu/smallsat/2017/all2017/91/

2. Inatani, Y., and Ohtsuka, H., "SS-520 Nano Satellite Launcher and its Flight Result," 32nd Annual Small Satellite Conference, SSC18-IX-03, August 2018. https://digitalcommons.usu.edu/smallsat/2018/all2018/308/

3. Barber, T.J., and Cowley, R.T., "Initial Cassini Propulsion System In-Flight Characterization," American Institute of Aeronautics and Astronautics, AIAA-2002-4152, Joint Propulsion Conference, July 2002. https://arc.aiaa.org/doi/10.2514/6.2002-4152

For References 4 through 23, please see the long version of this paper on the MEPAG website. https://mepag.jpl.nasa.gov/reports/decadal2023-2032/WhiteheadSmallerMAV2020Aug28.pdf 